\title{
LA PÉRDIDA DEL CARÁCTER TRANSCENDENTAL DE LA BELLEZA EN EL DE PULCHRO DE AGOSTINO NIFO
}

\author{
M.. Socorro Fernández García \\ Universidad de Burgos
}

\section{RESUMEN}

En este artículo se estudia la transformación del concepto de Belleza en la obra de Agostino Nifo. Para ello compararemos las princiales tesis defendidas por Nifo con las que defiende Santo Tomás de Aquino en esta materia. El resultado es el siguiente: los aspectos referidos a la Belleza en la obra de Nifo, están presentes en la tradición, pero vacíos de contenido. Como quiera que la Belleza es reducida al ámbito sensible, por eso pierde el carácter transcendental.

Palabras clave: Belleza, Nifo. Tomás de Aquino, transcendental

\section{ABSTRACT}

The loss of the transcendental character of beauty in Agostino Nifo's «De puchro». This article studies the transformation of the concept of Beauty in Nifo's work. For that purpose the most relevant thesis of his work De pulchro are being compared with the most significant writings of the work of Thomás d'Aquino. The results of this study lead us to the following conclusion: In Nifo's work we find the elements related to Beauty, that are present in the Tradition, but empty of content. As Beauty is reduced to the sensitive ambitus, it losses its transcendental character.

Key words: Beauty, Nifo, Thomas d'Aquino, Transcendental

\section{INTRODUCCIÓN}

Es importante, para valorar la obra de Nifo, atender al crédito que le prestaron sus contemporáneos y las generaciones sucesivas. Es significativo el hecho de que sus obras filosóficas se sucedieran sin interrupción en su vida y durante los 50 años que siguieron a su muerte. Nifo impartió su magisterio en las más diversas universidades: Padua, Salerno, Roma, Bolonia y Pisa. Gozó de prestigio en la corte; Carlos V lo hizo grande de España y el papa León X lo apreció mucho, hasta el punto de que en un motu proprio le exoneró de toda censura, le permitió insertar en su escudo las armas de la casa de Médicis y lo nombró conde palatino. Es indudable que su actividad como cortesano, compaginada con su dedicación a la docencia y a la investigación, le constituyen en un claro precursor del intelectual de la modernidad. 
Traductor y comentarista de Aristóteles, Averroes y Ptolomeo. Polemista, su imaginación, siempre atada por la razón, se debate entre sus grandes temas: la magia y los demonios, la toma de postura sobre la cuestión de la astrología y el debate filosófico-teológico sobre la inmortalidad. ${ }^{1}$

Su tratado sobre la belleza hay que encuadrarlo en la faceta de Nifo moralista. En este sentido, es conocido que un buen número de sus obras se dirigen al príncipe, al hombre y a la mujer de la corte. Garin, no muy benévolo con Agostino, dirá al respecto que parte de la fama que le rodeó en vida, perduró sólo gracias al carácter de grades figuras de hombres que aduló, calumnió o combatió a muerte. ${ }^{2}$

En su obra pretende conjugar rigor y método. El tratado avanza en su discurso, mediante una suerte de pequeñas tesis escolares, numeradas y precedidas de un enunciado, tal como lo hace Ficino en su tratado De amore. Hecho a trozos con las herramientas de la filosofía más pura, y a trozos con otros utensilios de la erudición clásica, se convierte así en una suerte de breviario o compendiosa lección en torno a todo lo que sobre la belleza y el amor se ha dicho. ${ }^{3}$ Garin sostendrá que es frecuente que en la obra de Nifo se articule una vasta erudición con una gran debilidad especulativa. $^{4}$

No vamos a entrar aquí a discutir la calidad científica de la obra de Nifo, ya que el propósito de este estudio no es otro que el de analizar la evolución que ha sufrido el concepto de belleza. En este sentido, es muy válido su tratado, ya que nos permite contemplar de un modo general no sólo la opinión de Agostino sino el sentir de una época, porque, en la medida en que Nifo pretende ofrecer un panorama completo de todo lo que se ha pensado en torno a la belleza, nos facilita estudiar la evolución del pensamiento respecto a la época anteror.

\section{LA BELLEZA EN AGOSTINO NIFO}

Soccas divide los 71 capítulos del tratado De pulchro en 10 apartados temáticos: a) La realidad de la belleza (cap. 1-5); b) aproximación negativa al concepto de belleza (cap. 6-11); c) crítica y discusión de las doctrinas neoplatónicas (cap. 12-26); d) exposición de la doctrina peripatética (cap. 27-33); e) las praeparationes o requisitos de la belleza (cap. 34-40); f) las diferencias entre platónicos y peripatéticos (cap. 41-53); g) Quaestiones amatoriae (cap. 54-63); h) la sensualidad de la belleza (cap. 64-65); i) teoria del pudor y de la decencia (cap. 66-69); y j) catálogo de mujeres y varones famosos por su belleza (cap. 70-71). ${ }^{6}$

1 A. Nifo, Sobre la belleza y el amor, trad. de Francisco Socas, Sevilla, Universidad de Sevilla, 1990, p. 10.

2 E. Garin, La revolución cultural del Renacimiento, trad. de Domènec Bergadà, Barcelona, Crítica, 1981, p. 172.

3 Nifo, o.c., cit. p. 22.

4 E. Garin, o.c., p. 172.

5 Para referirme a este tratado voy a seguir la traducción realizada por Francisco Socas, a la que ya he hecho alusión en notas anteriores. Citaré el tratado por el título latino, seguido del número romano del capítulo correspondiente.

6 Nifo, o.c., pp. 29-31. 
Entre otras tesis que se pueden destacar del tratado de Agostino, es muy relevante que para él la belleza se encuentra sobre todo en la naturaleza y no hay más belleza que la humana. ${ }^{7}$ Esto es así por la relación tan estrecha que existe entre la belleza y el amor, entendidos en términos de deseo. Es hermoso aquello que tiene como cualidad propia el atraer. ${ }^{8}$ Por lo tanto, no sólo no hay más belleza que la humana, sino que no hay más belleza que la corporal y sensible, aunque puntualiza que en el hombre se puede hablar de belleza del cuerpo, que es su «apostura» y belleza del alma, que su discreción. ${ }^{9} \mathrm{Al}$ igual que el amor se dice con propiedad del apetito sensitivo, lo bello en sí es algo que depende de los sentidos. ${ }^{10}$ Lo sensible es lo único real, porque el único amor real es también el sensible. Habla del amor como de un sentimiento que reside en el apetito sensitivo y que invita, halaga y arrastra nuestra alma al disfrute sensual de la belleza en sí, por medio de todos los sentidos, que son los que transmiten la imagen de lo bello hasta el alma. ${ }^{11}$

$\mathrm{Si}$ la belleza es algo sensible, es lógico que ninguna cosa simple sea bella. ${ }^{12} \mathrm{Nifo}$, siguiendo a los peripatéticos, relaciona la belleza con los sentidos y la verdad con lo inteligible. Además, lo bello es sólo para los sentidos humanos. ${ }^{13}$ «Hermoso será aquello que, merced a su propio encanto, nos arrastra hacia ese deseo que es sentimiento del apetito sensitivo y que está lleno de afanes y miedos». ${ }^{14}$ Este punto enlaza con una cuestión importante acerca de la objetividad de la belleza; es un hecho que no todos tienden a disfrutar de lo mismo, y la razón estriba precisamente en el carácter individual de quien percibe. ${ }^{15}$

De acuerdo con estos planteamientos, los requisitos o partes integrantes de la belleza es, por lo que se refiere al alma, la discreción, mientras que por lo que se refiere al cuerpo hay tantos requisitos como sentidos que trasladan la imagen de la belleza hasta el alma: la buena figura, que se relacionaría con la vista (a su vez, la buena figura implica: el orden, la medida y el aspecto); la armonía, relacionada con el oído; la fragancia, con el olfato; la blandura, con el tacto; y el dulzor, con el gusto. ${ }^{16}$

Nifo no omite en su tratado una comparación entre platónicos y aristotélicos. Para los platónicos, la belleza es una suerte de encanto que mueve al alma hacia el disfrute de la cosa bella; distinguen dos tipos de belleza: la intelectual y la vital. La belleza intelectual es la que, a través de la mente, mueve el alma hacia un disfrute de naturaleza intelectual. Dios, el ángel y el alma son bellos merced a este género de belleza. La belleza vital es la que a través de la vista o el oído, o ambos a la vez, mueve el alma a un disfrute de naturaleza vital, que halla su cumplimiento en la visión, la audición o ambas operaciones a la vez. Los peripatéticos añaden una tercera clase de belleza, la cor-

7 De pulchro, IV y V.

8 De pulchro, XIII, XVIII y XXXII.

9 De pulchro, V.

10 De pulchro, XXV.

11 De pulchro, XXX.

12 De pulchro, XXVI.

13 De pulchro, XXVII y XXVIII.

14 De pulchro, XXXI.

15 De pulchro, XLIX.

16 De pulchro, XXXV. 
pórea, que mueve el alma, a través de todos o varios de los sentidos, hacia un disfrute de naturaleza corporal que halla su cumplimiento en el abrazo y el contacto. Por otra parte, para éstos el asiento de la belleza corpórea es el género humano. Los seres de naturaleza intelectual deben considerarse buenos pero no bellos. Los seres corpóreos no son bellos tampoco. Sólo pueden decirse bellos aquellos serës que pueden ser objeto de amor de deseo: éstos son los humanos. ${ }^{17}$

Por último, se puede destacar también que para Nifo el deseo de disfrutar de la belleza es algo que se da por naturaleza, ${ }^{18}$ y virtudes como la discreción, el pudor y la decencia constituyen elementos importantes de la belleza. ${ }^{19}$

\section{LA BELLEZA COMO TRANSCENDENTAL}

Una de las primeras afirmaciones que sorprenden en Nifo es la reducción de la belleza al ámbito de lo humano. En esto no parece haber tenido en cuenta la tradición, pues es un dato histórico que en el pensamiento griego, desde Platón hasta Plotino, la belleza siempre se relacionó con el ente 0 con alguna de sus propiedades. ${ }^{20}$ En este punto es donde se puede apreciar mejor cómo en el tratado de Nifo se encuentran los elementos acerca de la belleza que están presentes en la tradición, pero vaciados de contenido. Al reducir la belleza al ámbito de lo sensible, los aspectos objetivo y subjetivo se problematizan, los requisitos o condiciones de la belleza se diversifican, y la relación de la belleza con la bondad y la verdad de las cosas pierde su unidad.

Es importante, para enjuiciar adecuadamente las tesis de Agostino, recordar la concepción tradicional de belleza. No es posible realizar el presente trabajo un recorrido a lo largo de la historia para ver cómo se ha tratado la cuestión de este transcendental; por tal motivo, recurro a la obra de Tomás de Aquino. La postura aquiniana sintetiza la tradición y marca el horizonte especulativo del pensamiento occidental. Aunque el aquinate propiamente no escribió ningún tratado De pulcrho, en sus obras están los elementos necesarios y suficientes para hacernos cargo de cuál es su pensamiento y, por consiguiente, para apreciar las diferencias respecto a la doctrina de Nifo. ${ }^{21}$

En un espigueo rápido por los textos más significativos del Aquinate, recordamos en primer lugar cómo la belleza y la bondad se equiparan: «En un sujeto determinado, la belleza y la bondad son una misma cosa, pues se fundan en una misma realidad, que es la forma, y por esto lo bueno se considera como bello. No obstante, difieren sus conceptos, porque el bien propiamente se refiere al apetito, ya que bueno es lo que todas las cosas apetecen, y, por tanto, debido a que apetito es una especie de movimiento hacia las cosas, tiene razón de fin. En cambio, lo bello se refiere al poder cognoscitivo, pues se llama bello aquello cuya vista agrada, y por esto la belleza consiste en la debida proporción, ya que los sentidos se deleitan en las cosas debidamente proporcionadas como en algo semejante a ellos, pues los sentidos, como toda facultad cognoscitiva son de algún

17 De pulchro, XLI.

18 De pulchro, XLVIII.

19 De pulchro, LXVI, -LXIX.

20 E. Forment, «La transcendentalidad de la belleza», Thémata. Revista de Filosofia, 9 (1992), p. 167.

21 E. de Bruyne, Etudes d'esthétique médiévale, III, Brujas, 1946, pp.278-315. 
modo entendimiento. Si pues el conocimiento se realiza por asimilación, y la semejanza se basa en la forma, lo bello pertenece propiamente a la razón de causa formal»». ${ }^{22}$

La belleza aparece en primer lugar como una propiedad del real. Significa el ser en tanto que resplandece para la inteligencia y para el apetito que es connatural con él. No está en función sólo del apetito sino del apetito, y de la inteligencia unidos. No es lo que todos apetecen, sino lo que agrada al ser contemplado. El fin no es la posesión sino el agrado. Esto implica que los sentidos más aptos para percibir la belleza son aquellos que son más cognoscitivos, como la vista o el oído. Aunque la belleza no depende de los sentidos sino de aquella propiedad que tienen las cosas para que agraden al ser contempladas. Esta propiedad añade un cierto orden a la potencia cognitiva, de tal modo que, así como se llama bien a todo lo que agrada en absoluto al apetito, se llama bello a aquello cuya aprehensión nos complace. ${ }^{23}$

Se puede apreciar que la belleza, como aspecto del ser, en su origen ontológico, queda independiente del poder constituyente de la inteligencia humana, aunque implica constitutivamente una relación interior del ser al espíritu. Se puede hablar, por lo tanto, de condiciones ontológicas de la belleza, es decir, si todas las cosas tiene ser y no todas se dicen bellas, algo habrá que determine en las cosas la razón de belleza. Recordamos: «Pues para que haya belleza se requieren tres condiciones: primero, la integridad o perfección; lo inacabado es por ello feo; segundo, la debida proporción y armonía; y, por último, la claridad». ${ }^{24}$ Por otro lado, el espíritu constituye a su vez la condición subjetiva de la belleza, pues ésa no se acaba en su despliegue sino en el interior del espíritu.

Para Tomás de Aquino, las tres condiciones ontológicas son propiedades de la forma. La belleza está fundada en la forma y consiste en el esplendor de ella para la inteligencia. De un modo más preciso: la perfección y la proporción son movimientos inmanentes de la forma hacia su propio acabamiento, que es la actualidad de su unidad. La consecuencia de este movimiento de unificación interior es la claridad, es decir, el resplandor de la inteligibilidad de la forma para una inteligencia; y en otros terminos también, el resplandor revelador de la verdad del ser. Esta claridad es el constitutivo formal de la belleza, su diferencia específica respecto a la bondad. ${ }^{25}$

Para santo Tomás la belleza constituye una propiedad transcendental que unifica la bondad y la verdad, pero con la bondad como materia y el resplandor de la verdad como la forma. La belleza se acaba, por lo tanto, en primer lugar en la inteligencia y después en el corazón, entendido como el lugar del movimiento apetitivo propio de la inteligencia donde ella engendra una alegría.

No se puede olvidar que la distinción entre los transcendentales es una distinción de razón que corresponde a una distinción virtual en el ser. En la realidad no se puede separar la verdad de la bondad. En este sentido, se puede afirmar que la belleza debe ser concebida como el resplandor de la verdad y de la bondad unificadas en el ser; por lo tanto, también se puede presentar simplemente como el resplandor del ser. Es decir, esto puede comprenderse como el resplandor de la verdad

22 Tomás de Aquino, S. Th., I, q. 5. a. 4, ad. 1; y también. S.Th., I-II, q. 27, a. 1, ad. 3.

23 Tomás de Aquino, S. Th., I-II, q. 27, a. 1, ad. 3.

24 Tomás de Aquino, S. Th., I. q. 39, a. 8, in c.

25 P. Dasseleer, «L'être et la beauté selon Saint Thomas d'Aquin», en J. Follon y J. McEvoy, Actualité de la pensée mediévale, Lovaina-París, Peeters, 1994, pp. 272-273. 
y del bien unificados, aventajándolos y corrigiéndolos el uno por el otro, como el acto total y final del ser donde todos los transcendentales se compenetran en un resplandor último. ${ }^{26}$

Por otro lado, si sostenemos la existencia de un orden en los transcendentales, y la belleza tiene que ver con la verdad y con el bien, la precedencia de la verdad sobre el bien justifica en parte que la belleza se incline más desde el lado de la verdad y de la inteligencia. ${ }^{27}$

La unidad de los transcendentales aplicada al hombre nos conduce a afirmar que la auténtica belleza humana no es la sensible, sino la que de deriva de su espíritu, porque lo que constituye metafísicamente al hombre no es su cuerpo sino su alma, en unidad substancial con su cuerpo. De nuevo el Aquinte nos proporciona el elemento que clarifica la cuestión, al sostener que en el hombre la belleza se identifica con la honestidad, que es su bondad, aspecto que transciende el ámbito de su corporalidad, pues se trata de una proporción entre las obras y el espíritu. ${ }^{28}$

Por otro lado, Tomás de Aquino no desprecia la dimensión subjetiva «Pulchra enim dicuntur quae visa placent».$^{29}$ Según el Doctor común, la belleza de un ser se reconoce subjetivamente por la alegría que suscita en el corazón, con ocasión de su contemplación por la inteligencia; a ella le pertenece esencialmente aquietar el apetito connatural a la inteligencia; de este modo, la alegría que se experimenta con ocasión de la vista intuitiva de una forma es el signo subjetivo de la presencia de la belleza.

La naturaleza, en efecto, es bella tan sólo con la condición de que sea comprendida, y lo que hay que comprender es el orden que en ella resplandece. Cuando el hombre percibe alguna partícula de este orden, cuando sorprende en un ser o en un grupo de seres los resplandecientes caminos por los cuales realiza la naturaleza la variedad en la unidad, goza entonces de valor estético. ${ }^{30}$

La aprehensión de lo bello y el conocimiento estético, tiene la peculiaridad, frente a otro tipo de conocimiento, de que es capaz de captar lo inteligible sin prescindir de las condiciones sensibles. La inteligencia se goza con lo percibido porque capta la unidad, el esplendor de la forma, la armonía y la proporción.

Queda por decir de qué manera el espíritu constituye la condịción ontológica subjetiva de la belleza. La belleza es irradiación del ser para el espíritu; de esto resulta que ella no se acaba como belleza más que con la condición de una captación subjetiva. En este sentido, es importante tener en cuenta que, siendo la belleza convertible con el ser, al captar la belleza se captan los demás transcendentales, y en la medida en que la forma es reflejo de la inteligencia creadora, la captación de la belleza es la puerta que facilita el acceso desde el plano sensible al mundo del espíritu.

26 Ibiden, pp. 275-276.

27 Tomás de Aquino, De Veritate, q. 1, a. 1, in c., q. 21, a. 3, in c, S.Th., I, q. 16, a. 4, ad. 2; Cfr: Forment, o.c., pp. 176-177, y L. Polo, «Claves del nominalismo y del idealismo», Cuadernos de Anuario Filosófico, Pamplona, n. ${ }^{\circ} 5$ (1993), p. 8 .

28 Tomás de Aquino, S.Th., II-II, q. 145, a. 2, in c.

29 Tomás de Aquino, S.Th., I, q. 5, a. 4. ad. 1.

30 M de Wulf, Arte y belleza, Barcelona, Atlántida, 1950, p. 139. 
Lo que funda en último término la belleza finita, incapaz de fundarse a sí misma es la belleza del Creador. ${ }^{31} \mathrm{Si}$ ella existe hermosa y buena antes de que sea captada por el espíritu humano, si ella está ya acabada, es en virtud de su creación por la intuición de Dios, en virtud del conocimiento puramente activo y de la voluntad de Dios. Y si el espíritu humano es, sin embargo, condición subjetiva para el acabamiento de la belleza, sólo es bajo la relación según la cual es belleza para el hombre. El ser no irradia forzosamente para el espíritu humano más que si éste lo aprehende activamente. Pero para tal hecho este espíritu debe, en primer lugar y finalmente, ser aprendido en su pasividad por una irradiación ya constituida. El ser es bello antes de toda aprehensión por el hombre, en el sentido de que el Espíritu absoluto de Dios es la condición subjetiva por excelencia del esplendor de lo real. ${ }^{32}$

\section{CONCLUSIONES}

Nifo ha captado la particularidad de la belleza respecto al bien y a la verdad. Ha captado también la relación que tiene con el amor. Sin embargo, en la medida en que reduce la belleza al ámbito de lo sensible y lo compuesto, y en la medida en que la define como cualidad que hace a las cosas más apetecibles, fractura la unidad de los transcendentales. La belleza queda encerrada en los límites del deseo, pierde su relación con la verdad y con el ente, y se limita a ser la cara externa del bien deleitable.

Nifo no llega a ver que la belleza no es sólo resplandor de la bondad del ser, en tanto que es comunicación de sí, como consecuencia de su movimiento inmanente de perfección y de proporción, comprendidos de modo dinámico. La belleza es también el resplandor de la verdad del ser, en tanto que es inteligible, del ser en tanto que revela su propia perfección y su proporción comprendidas estáticamente, y además, la belleza es también, de un modo más profundo, manifestación del ser en tanto que él es indiviso, de la unidad, que es el origen y el fin, la piedra angular de la perfección y de la proporción de lo real. Sólo la irradiación en tanto que tal (claritas y también splendor), constituye específicamente la belleza, ${ }^{33}$ Por lo tanto, aunque nuestra captación de la belleza pasa por lo sensible, que es lo que nos resulta más asequible, no se puede sostener que a lo inteligible no le corresponde en propiedad el ser bello.

La belleza, que es el esplendor (irradiación) del ser como unidad del bien y de la verdad, se dirige al espíritu, que es là unidad de las dos facultades, la intelectiva y la apetitiva. El orden del conocer es fundamentalmente aprehensión y posesión espiritual del ser, es decir, aprehensión y posesión por el intelecto y por el apetito indisolublemente unido al intelecto. Pero el orden del juicio cordial es también aprehensión y posesión espiritual del ser, es decir aprehensión y posesión por el apetito y por la inteligencia indisolublemente unida al apetito.

31 Adrationi pulchri, sive decori, concurrit et claritas et debita proportio: dicit enim quod Deus dicitur pulcher sicut universorum consonantiae et claritas causa, Tomás de Auino, S.Th., II-II, q. 145, a. 2, in c.

32 Dasseleer; o.c., p. 286.

33 Ibiden, p. 281. 
Por el contrario, Nifo, al considerar la belleza como esplendor del bien por encima de su relación con la verdad, hace hincapié en el aspecto volitivo, entendido como deseo, sobre el intelectual. La belleza ya no es lo que visto agrada, sino lo que hace a algo deseable a los sentidos. Es lógico que para Agostino el tacto, el gusto y el olfato se sitúen al mismo nivel que los otros sentidos más intelectuales; de hecho, lo que capta la vista y el oído ya no transciende el plano sensible, pues el orden, la medida y el aspecto, a los que se refiere Nifo, corresponden a la figura, accidente cualidad, no a la forma substancial del ente.

M. Socorro Fernández García

Facultad de Humanidades y Educación

Universidad de Burgos

Villadiego $s / n$.

E- 09001 BURGOS 